\title{
MODELAMENTO MATEMÁTICO DA LONGEVIDADE DE SEMENTES DE CAMU-CAMU
}

\author{
Roberto Sinício ${ }^{1}$
}

\section{RESUMO}

O objetivo deste trabalho foi obter um modelo matemático da longevidade de sementes de camu-camu (Myrciaria dubia (H.B.K.) McVaugh). Dados experimentais da bibliografia referentes às curvas de sobrevivência de sementes de camu-camu, armazenadas sob teores de água e temperaturas, foram utilizados em regressões lineares e não lineares para determinação dos coeficientes de diferentes modelos de longevidade. Os coeficientes dos modelos recomendados foram determinados usando-se os valores das perdas da germinação e emergência, da germinação e emergência iniciais, da temperatura, do teor médio de água das sementes e do período de armazenagem, para todas as curvas de sobrevivência. O modelo modificado de probit foi recomendado porque resultou no melhor ajuste para 57 observações experimentais, quando comparado com outros três modelos, tanto para a perda da germinação $\left(\mathrm{R}^{2}\right.$ de 0,90 , desvio padrão de $12,3 \%$ e grau de significância dos coeficientes do modelo menor que $0,4 \%$ de probabilidade de erro no teste t), como para a perda da emergência $\left(\mathrm{R}^{2}\right.$ de 0,87 , desvio padrão de $13,3 \%$ e grau de significância dos coeficientes do modelo menor que $0,1 \%$ de probabilidade de erro no teste $t$ ).

Palavras-chave: Myrciaria dubia (H.B.K.) McVaugh, viabilidade, previsão, armazenamento, qualidade

\section{ABSTRACT \\ MATHEMATICAL MODELLING OF THE LONGEVITY OF CAMU-CAMU SEEDS}

The objective of this work was to obtain a mathematical model of the longevity of camu-camu seeds (Myrciaria dubia (H.B.K.) McVaugh). Experimental data from the bibliography of the survival curves of camu-camu seeds, stored under different moisture contents and temperatures, were used in linear and non linear regressions for determining the coefficients of different longevity models. The coefficients of the recommended models were determined using the values of germination and emergence losses, initial germination and emergence, temperature, average moisture content of the seeds and the storage period for all survival curves. The modified probit model was recommended because it resulted in the best fits for the 57 experimental observations when compared with other three models, as well as for germination loss $\left(\mathrm{R}^{2}\right.$ of 0.90 , standard error of $12.3 \%$, and all model coefficients significant at $0.4 \%$ probability level in the $t$ test) and emergence loss ( $\mathrm{R}^{2}$ of 0.87 , standard error of $13.3 \%$, and all model coefficients significant at $0.1 \%$ probability level in the $t$ test).

Keywords: Myrciaria dubia (H.B.K.) McVaugh, viability, prediction, storage, quality

Recebido para publicação em 12/08/2013. Aprovado em 04/03/2015.

1 - Engenheiro de Alimentos, Ph. D., Pesquisador, Departamento de Fitotecnia-UFV, e-mail: rsinicio@ufv.br 


\section{INTRODUÇÃO}

O camu-camu (Myrciaria dubia (H.B.K) McVaugh) é uma espécie frutífera natural da Amazônia pertencente à família Myrtaceae, encontrada em áreas sujeitas a inundações, como às margens dos rios, lagos, regiões de várzeas e igapós (McVAUGH, 1963; SILVA, 2001). O camu-camu tem despertado interesse em diversos países pelo seu potencial de produção de ácido ascórbico (YUYAMA et al., 2002), uma vez que a concentração desse ácido é maior do que na maioria das plantas cultivadas (VILLANUEVATIBURCIO et al., 2010). O período de semeadura do camu-camu é restrito, podendo existir rápida perda de sua capacidade germinativa dependendo da umidade da semente. Ainda há poucas pesquisas sobre a manutenção de sua viabilidade por longos períodos de tempo durante o armazenamento (FERREIRA; GENTIL, 2003). A sensibilidade ao dessecamento é um dos fatores relacionados à rápida perda de viabilidade das sementes de camucamu por não tolerarem o dessecamento e umidade abaixo de 20\%, enquadrando-se, portanto, na categoria de sementes recalcitrantes (SACANDÉ et al., 2004). A dificuldade de conservação das sementes concorre para a instabilidade na produção de mudas e para a desestabilização dos estoques em bancos de germoplasma (GENTIL et al., 2004).

Admitindo-se que sensibilidade à dessecação interfere na conservação dessas sementes (GENTIL; FERREIRA, 2000), logo, sugere-se a manutenção do grau de umidade elevado, pelo armazenamento em água (FERREIRA; GENTIL, 2003; YUYAMA et al., 2011) ou em embalagem de polietileno, a $20^{\circ} \mathrm{C}$, após breve secagem natural à sombra (GENTIL; FERREIRA, 1991). Gentil et al. (2004) constataram que as sementes de camu-camu têm a conservação favorecida pela associação do grau de umidade de $43 \%$ com a temperatura de armazenamento de $10^{\circ} \mathrm{C}$.

As sementes recalcitrantes, além de incapazes de suportar adequadamente a dessecação abaixo de teores críticos de água, estão sujeitas ao rompimento de células, por expansão de volume, ao serem armazenadas sob temperatura de congelamento do conteúdo celular hidratado. Adicionalmente, em espécies tropicais, essas sementes podem ser termicamente injuriadas em temperaturas entre 10 e $15^{\circ} \mathrm{C}$ (HONG et al., 1996). Essas exigências, para as condições de armazenamento, contrapõem-se às indicadas para as sementes ortodoxas, as quais, fundamentalmente, demandam baixos teores de água e de temperatura para a adequada manutenção da qualidade (YUYAMA et al., 2011).

As sementes ortodoxas são as que podem ser desidratadas a valores muito baixos de água (entre $5 \%$ e $7 \%$ deteordeágua), semperderema viabilidade. A longevidade dessas sementes, dependendo das espécies, aumenta progressivamente com a redução do teor de água e o armazenamento em baixas temperaturas (SACANDÉ et al., 2004). As sementes ortodoxas são tolerantes à dessecação, por tolerarem os efeitos imediatos da perda severa de água. Conforme Black et al. (2002), a tolerância das sementes à dessecação pode ser definida como a capacidade de recuperação das funções biológicas após desidratação até a condição de que não reste fase líquida nas células (por exemplo, teor de água de $5 \%$ ou menos da massa seca, em equilíbrio com o potencial hídrico de $-200 \mathrm{MPa}$ ou menos). A longevidade das sementes armazenadas aumenta progressivamente à medida que a UR do ambiente de armazenamento é reduzida até, aproximadamente, $20 \%$ e, então, uma tendência inversa pode ser observada com UR abaixo desse valor (MEDEIROS; EIRA, 2006).

Os modelos matemáticos usados para descrever a perda da viabilidade de sementes ortodoxas durante o armazenamento, geralmente, consideram a viabilidade inicial, período de armazenagem, teor de água e temperatura das sementes (ELLIS; ROBERTS, 1980; GINER et al., 1991; SOKHANSANJ, 1997). O modelo desenvolvido por Ellis e Roberts (1980) é do tipo probabilístico, enquanto os modelos utilizados por Giner et al. (1991) e Sokhansanj (1997) são do tipo exponencial.

O modelo de probit de Ellis e Roberts (1980) tem sido usado com sucesso para predizer a perda da germinação de sementes ortodoxas durante o período de armazenamento. Afonso Junior et al., (2000) concluíram, entretanto, que o modelo exponencial foi o que melhor se ajustou aos dados experimentais para predizer o potencial germinativo de sementes de soja da variedade Santa Rosa durante o armazenamento. Os coeficientes do modelo de Ellis e Roberts (1980) já foram determinados, por alguns pesquisadores, para mais de 77 cultivares, de 48 espécies (ELLIS; ROBERTS, 1980; ELLIS et al., 1982; HONG et al., 1996; SINÍCIO et al., 2008, 2009; SINÍCIO, 2011; SINÍCIO, 2013).

Considerando-se que não se encontrou na bibliografia consultada nenhum estudo sobre o equacionamento da perda da viabilidade de sementes de camu-camu armazenadas, objetivou-se com este trabalho elaborar um modelo matemático para predizer a longevidade de sementes da espécie em questão.

\section{REVENG} 203-211p. 


\section{MATERIAL E MÉTODOS}

Os dados experimentais obtidos por Gentil (2003) na conservação de sementes de camucamu (Myrciaria dubia (H. B. K.) McVaugh) foram usados em regressões lineares e não lineares para determinação dos coeficientes de diferentes modelos de longevidade. Os dados utilizados consistem em perdas da germinação e emergência de plântulas, da germinação e emergência iniciais, da temperatura, do teor médio de água e do período de armazenagem, para todas as curvas de sobrevivência das sementes armazenadas, sob teores de água $(48,43,40,34,24,18$ e $14 \%$ b.u. $)$ e temperaturas $\left(10,20\right.$ e $\left.30^{\circ} \mathrm{C}\right)$, durante 280 dias.

Primeiramente testou-se, como referência, o modelo de probit de Ellis e Roberts (1980), assumindo-se como viabilidade os valores da germinação e emergência de plântulas, transformados em probit, conforme segue

$$
\begin{aligned}
& v=K i-p / \sigma \\
& \sigma=10^{\left(K E-C W \log m-C H t-C Q t^{2}\right)}
\end{aligned}
$$

em que,

$v$ - viabilidade (germinação e emergência de plântulas) das sementes (probit);

$K i=v_{0}=$ viabilidade inicial (germinação $\mathrm{e}$ emergência iniciais) das sementes (probit);

$p$ - período de armazenamento (dias);

$\sigma=$ desvio padrão da freqüência de distribuição

das sementes mortas durante o período de armazenamento (dias);

$m=$ teor de água ( $\%$, base úmida);

$t=$ temperatura $\left({ }^{\circ} \mathrm{C}\right)$;

$K E, C W, C H$ e $C Q=$ coeficientes específicos para cada espécie, independentes do genótipo e das condições de pré-estocagem.

Em sequencia, testou-se o seguinte modelo modificado de Ellis e Roberts (1980):

Também foram testados os seguintes modelos, similares aos utilizadas por Giner et al. (1991) e Sokhansanj (1997), respectivamente:

Regressões lineares múltiplas foram utilizadas em todos os modelos, após a sua linearização pela aplicação de logaritmos, apenas para a estimativa dos valores iniciais a serem usados em regressões não lineares. Esse procedimento se justificou porque os modelos linearizados com o uso de logaritmos apresentam distribuição simétrica de erros para o logaritmo da função desejada, mas assimétrica para a função original, o que dá uma estimativa da mediana da distribuição dos erros e não da média dos erros (SAPRA et al., 2003). A obtenção de melhores ajustes para modelos não linearizados usando-se regressões não lineares foi confirmada para o equacionamento da longevidade de sementes de café (SINÍCIO, 2011). As rotinas computacionais apresentadas por Press et al. (1990) para fazer as regressões não lineares, pela técnica dos mínimos quadrados, foram utilizadas. A escolha do melhor modelo foi feita com base no coeficiente linear de regressão ajustado $\left(\mathrm{R}^{2}\right)$, no desvio padrão (d.p.) dado em percentagem de germinação ou emergência de plântulas e na praticidade do uso do modelo.

$$
\begin{aligned}
& v=v_{0}-p / 10^{\left(f_{0}+f_{1} m+f_{2} m^{2}+f_{3} m^{3}+t\left(f_{4} m+f_{5} m^{2}+f_{6} m^{3}+f_{7} t\right)\right)} \\
& V=V_{0} \cdot 10^{\left[-p \cdot 10^{\left(g_{0}+g_{1} m+g_{2} m^{2}+g_{3} m^{3}+t\left(g_{4} m+g_{5} m^{2}+g_{6} m^{3}+g_{7} t\right)\right)}\right]} \\
& V=V_{0}-p \cdot 10^{\left(h_{0}+h_{1} m+h_{2} m^{2}+h_{3} m^{3}+t\left(h_{4} m+h_{5} m^{2}+h_{6} m^{3}+h_{7} t\right)\right)}
\end{aligned}
$$

em que,

$V=G$ (germinação) e $E$ (emergência) das sementes (\%);

$V_{0}=G_{0}$ (germinação inicial) e $E_{0}$ (emergência inicial) das sementes (\%);

$f_{i}, g_{i}$ e $\left.h_{i} \mathrm{i}=0,1,2 \ldots \mathrm{n}\right)=$ coeficientes específicos para cada espécie e variedade de semente. 


\section{RESULTADOS E DISCUSSÃO}

Dentre os quatro modelos comparados para previsão da perda da germinação das sementes de camu-camu armazenadas, o de Ellis e Roberts (1980) foi o que apresentou os maiores erros no ajuste, com $\mathrm{R}^{2}$ de 0,79 e d.p. de $18,0 \%$ (Quadro 1). Para previsão da perda da emergência, entretanto, os maiores erros foram obtidos com o modelo modificado de Sokhansanj (1997), com $\mathrm{R}^{2}$ de 0,84 e d.p. de $15,0 \%$. Os coeficientes $C T$ do modelo de Ellis e Roberts (1980) para germinação e emergência, assim como o coeficiente $C W$ para emergência (Quadro 2), resultaram em valores positivos, ao contrário daqueles normalmente obtidos para sementes ortodoxas (ELLIS; ROBERTS, 1980; ELLIS et al., 1982; HONG et al., 1996).

O modelo modificado de Ellis e Roberts (1980), comparado com os outros modelos (Quadro 1), resultou em melhor ajuste tanto para a previsão da perda da germinação, com $\mathrm{R}^{2}$ de 0,90 e d.p. de $12,3 \%$, quanto para a previsão da perda da emergência, com $\mathrm{R}^{2}$ de 0,87 e d.p. de $13,3 \%$. Um modelo modificado de Ellis e Roberts (1980), semelhante ao testado para sementes de camucamu, também resultou no melhor ajuste para previsão da perda da germinação de sementes de jabuticaba armazenadas, com $\mathrm{R}^{2}$ de 0,87 e desvio padrão de 12,3\% (SINÍCIO, 2013).

O modelo modificado de Giner et al. (1991) resultou no segundo pior resultado para a previsão da perda da germinação de sementes de camucamu armazenadas (Quadro 1). Este modelo, entretanto, resultou no segundo melhor resultado para previsão da perda da emergência, com $\mathrm{R}^{2}$ de 0,86 e d.p. de 14,2 .
Os valores dos coeficientes obtidos nas regressões não lineares, quando comparados para prever perdas da germinação e emergência utilizando um mesmo modelo matemático (Quadro 2), têm o mesmo sinal e, aproximadamente, a mesma ordem de grandeza, com exceção do coeficiente $C W$. O grau de significância de todos os coeficientes dos modelos foi menor que $0,1 \%$ de probabilidade de erro no teste $t$, com exceção dos coeficientes $f_{0}$ a $f_{7}$ para germinação, cujo grau de significância foi menor que $0,4 \%$ (Quadro 2). Os baixos valores dos níveis de significância obtidos para todos os coeficientes indicam alto grau de confiança nas suas estimativas e adequada convergência dos modelos no processo iterativo utilizado.

Os resultados obtidos com o modelamento matemático das perdas da germinação e emergência de sementes de camu-camu armazenadas parecem indicar que cada espécie de semente recalcitrante tem comportamento peculiar, tornando-se, assim, difícil desenvolver um modelo generalizado para a previsão da longevidade destas espécies. Esses resultados justificam a necessidade de se comparar diferentes modelos matemáticos para outras espécies de sementes recalcitrantes a serem estudadas.

Considerando-se, portanto, os erros obtidos e a praticidade do uso da equação, recomenda-se utilizar o modelo modificado de Ellis e Roberts (1980) para predizer as perdas da germinação e emergência de sementes de camu-camu armazenadas para temperaturas variando de 10 a $30^{\circ} \mathrm{C}$ e teores de água de 23,3 a 49,4. Os gráficos dos resultados calculados e experimentais para as perdas da germinação e emergência das sementes de camu-camu armazenadas (Figuras 1 e 2) demonstram a razoável precisão do ajuste para o modelo recomendado.

Quadro 1. Coeficientes lineares de regressão ajustado e desvios padrões obtidos nas regressões não lineares dos modelos, para previsão das perdas da germinação e emergência de sementes de camu-camu armazenadas

\begin{tabular}{lcccc}
\hline \multirow{2}{*}{ Modelo } & \multicolumn{2}{c}{ Germinação } & \multicolumn{2}{c}{ Emergência } \\
\cline { 2 - 5 } & $\mathrm{R}^{2}(\%)$ & d.p. (\%) & $\mathrm{R}^{2}(\%)$ & d.p. (\%) \\
\hline Ellis e Roberts (1980) & 0,79 & 18,0 & 0,86 & 14,4 \\
Ellis e Roberts (1980) modificado & 0,90 & 12,3 & 0,87 & 13,3 \\
Giner et al. (1991) modificado & 0,85 & 15,2 & 0,86 & 14,2 \\
Sokhansanj (1997) modificado & 0,86 & 14,5 & 0,84 & 15,0 \\
\hline
\end{tabular}

${ }^{*}$ Os valores do $\mathrm{R}^{2}$ e d.p. foram calculados para a germinação e emergência em percentagem, após o seu cálculo em probit. 
Quadro 2. Valores dos coeficientes e respectivos desvios padrões obtidos nas regressões não lineares dos modelos, para previsão das perdas da germinação e emergência de sementes de camu-camu armazenadas* $^{*}$

\begin{tabular}{|c|c|c|c|c|}
\hline \multirow[b]{2}{*}{ Símbolo } & \multicolumn{2}{|c|}{ Germinação } & \multicolumn{2}{|c|}{ Emergência } \\
\hline & Coeficiente & Desvio padrão & Coeficiente & Desvio padrão \\
\hline$K E$ & 2,01410 & $1,6619 \times 10^{-1}$ & 1,0532 & $2,3002 \times 10^{-01}$ \\
\hline$C W$ & $-4,1660 \times 10^{-1}$ & $6,3837 \times 10^{-2}$ & $1,8502 \times 10^{-01}$ & $5,2288 \times 10^{-02}$ \\
\hline$C T$ & $1,2527 \times 10^{-1}$ & $1,3625 \times 10^{-2}$ & $1,4331 \times 10^{-01}$ & $2,1000 \times 10^{-02}$ \\
\hline$C Q$ & $-4,1025 \times 10^{-3}$ & $3,7424 \times 10^{-4}$ & $-4,6039 \times 10^{-03}$ & $5,7308 \times 10^{-04}$ \\
\hline$f_{o}$ & $3,9003 \times 10$ & $1,0446 \times 10$ & $2,9698 \times 10$ & 7,0287 \\
\hline$f_{1}$ & $-3,2054$ & 1,0360 & $-2,3758$ & $6,4228 \times 10^{-01}$ \\
\hline$f_{2}$ & $8,8391 \times 10^{-02}$ & $2,2429 \times 10^{-2}$ & $6,3640 \times 10^{-02}$ & $1,3172 \times 10^{-02}$ \\
\hline$f_{3}$ & $-7,9775 \times 10^{-04}$ & $2,1828 \times 10^{-4}$ & $-5,5616 \times 10^{-04}$ & $1,2114 \times 10^{-04}$ \\
\hline$f_{4}$ & $1,7277 \times 10^{-02}$ & $3,0269 \times 10^{-3}$ & $1,6790 \times 10^{-02}$ & $2,2394 \times 10^{-03}$ \\
\hline$f_{5}$ & $-5,4508 \times 10^{-04}$ & $1,1044 \times 10^{-4}$ & $-4,4215 \times 10^{-04}$ & $7,0564 \times 10^{-05}$ \\
\hline$f_{6}$ & $5,5693 \times 10^{-06}$ & $1,6735 \times 10^{-6}$ & $3,8401 \times 10^{-06}$ & $9,7332 \times 10^{-07}$ \\
\hline$f_{7}$ & $-5,5157 \times 10^{-03}$ & $1,5142 \times 10^{-3}$ & $-6,3319 \times 10^{-03}$ & $1,0389 \times 10^{-03}$ \\
\hline$g_{o}$ & $-3,0382 \times 10$ & 1,5136 & $-1,0051 \times 10^{+02}$ & 1,6579 \\
\hline$g_{1}$ & 2,8518 & $1,1273 \times 10^{-1}$ & 7,8585 & $1,2670 \times 10^{-01}$ \\
\hline$g_{2}$ & $-8,5720 \times 10^{-02}$ & $2,6662 \times 10^{-3}$ & $-2,0231 \times 10^{-01}$ & $2,9458 \times 10^{-03}$ \\
\hline$g_{3}$ & $8,0883 \times 10^{-04}$ & $2,2462 \times 10^{-5}$ & $1,7032 \times 10^{-03}$ & $2,4245 \times 10^{-05}$ \\
\hline$g_{4}$ & $-2,6049 \times 10^{-02}$ & $6,3405 \times 10^{-4}$ & $-1,6777 \times 10^{-02}$ & $4,3706 \times 10^{-04}$ \\
\hline$g_{5}$ & $9,0082 \times 10^{-04}$ & $3,0057 \times 10^{-5}$ & $3,3848 \times 10^{-04}$ & $1,6748 \times 10^{-05}$ \\
\hline$g_{6}$ & $-9,2541 \times 10^{-06}$ & $3,4031 \times 10^{-7}$ & $-2,3712 \times 10^{-06}$ & $2,1050 \times 10^{-07}$ \\
\hline$g_{7}$ & $6,0118 \times 10^{-03}$ & $1,2818 \times 10^{-4}$ & $8,3638 \times 10^{-03}$ & $2,1875 \times 10^{-04}$ \\
\hline$h_{o}$ & $-6,9433 \times 10$ & 1,4684 & $-2,6721 \times 10$ & 1,2475 \\
\hline$h_{1}$ & 5,6507 & $1,1608 \times 10^{-1}$ & 2,2034 & $1,0104 \times 10^{-01}$ \\
\hline$h_{2}$ & $-1,4880 \times 10^{-01}$ & $3,0915 \times 10^{-3}$ & $-5,8608 \times 10^{-02}$ & $2,4827 \times 10^{-03}$ \\
\hline$h_{3}$ & $1,2830 \times 10^{-03}$ & $2,6727 \times 10^{-5}$ & $5,1235 \times 10^{-04}$ & $2,0835 \times 10^{-05}$ \\
\hline$h_{4}$ & $-2,6455 \times 10^{-02}$ & $5,9667 \times 10^{-4}$ & $-1,6762 \times 10^{-02}$ & $4,8774 \times 10^{-04}$ \\
\hline$h_{5}$ & $9,0839 \times 10^{-04}$ & $2,5568 \times 10^{-5}$ & $4,7464 \times 10^{-04}$ & $2,0977 \times 10^{-05}$ \\
\hline$h_{6}$ & $-9,3594 \times 10^{-06}$ & $3,1342 \times 10^{-7}$ & $-4,3637 \times 10^{-06}$ & $2,2970 \times 10^{-07}$ \\
\hline$h 7$ & $6,2305 \times 10^{-03}$ & $7,9109 \times 10^{-5}$ & $5,8299 \times 10^{-03}$ & $1,0019 \times 10^{-04}$ \\
\hline
\end{tabular}

${ }^{*}$ O grau de significância de todos os coeficientes dos modelos foi menor que $0,1 \%$ de probabilidade de erro no teste $t$ com exceção dos coeficientes $f_{0}$ a $f_{7}$ para germinação cujo grau de significância foi menor que $0,4 \%$. 
$10^{\circ} \mathrm{C}$

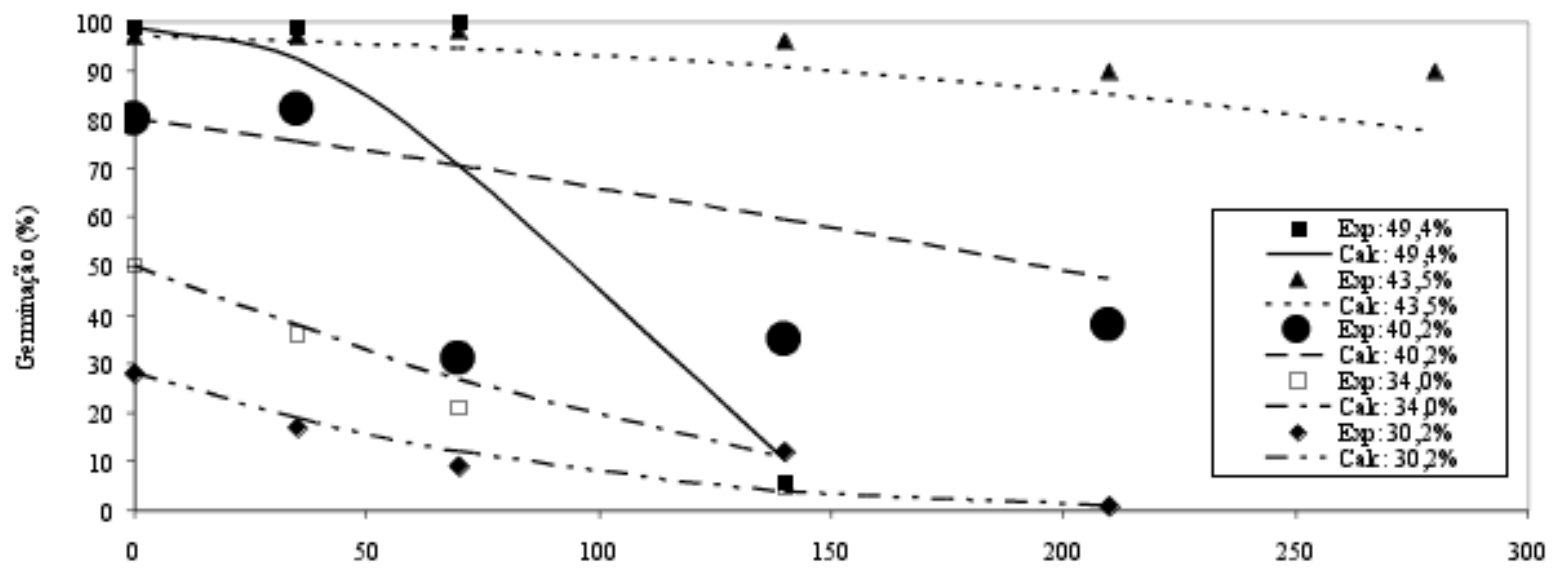

$20^{\circ} \mathrm{C}$

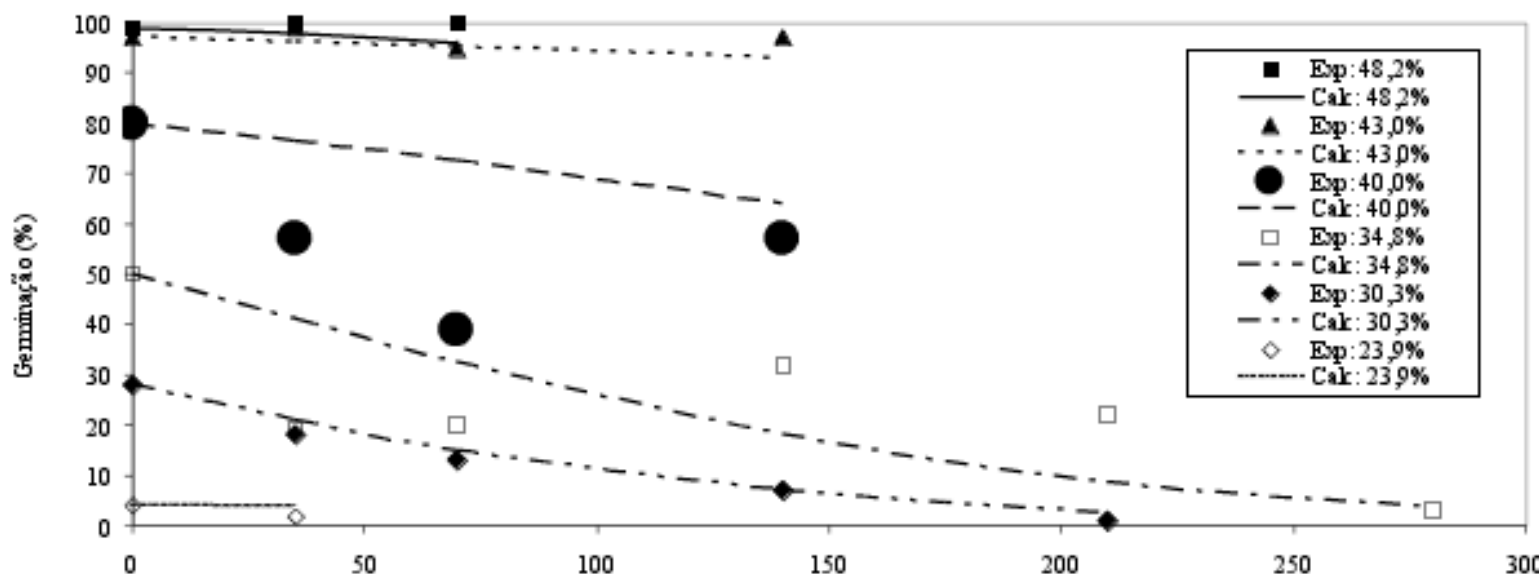

$30^{\circ} \mathrm{C}$

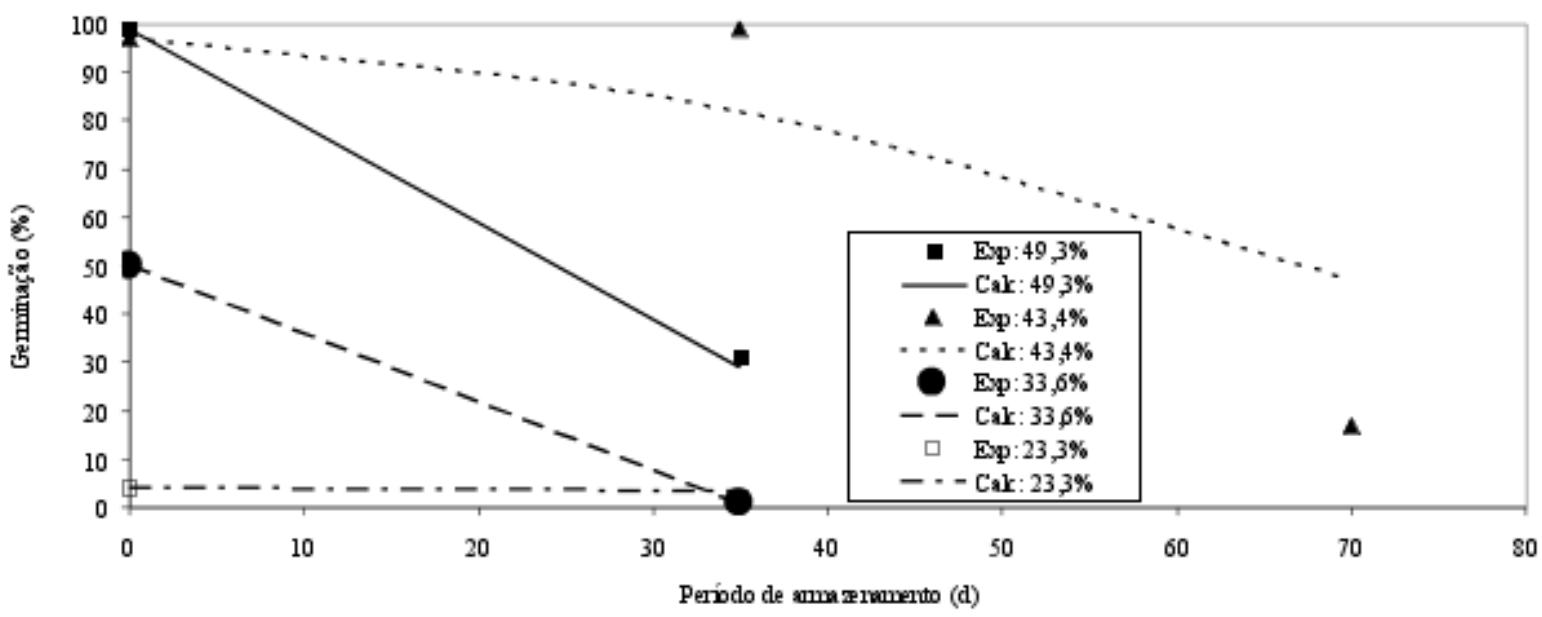

Figura 1. Valores experimentais e calculados da perda da germinação de sementes de camu-camu em função do período de armazenamento para três temperaturas e teores de água: (A) $10{ }^{\circ} \mathrm{C}$ e de 30,2 a $49,4 \%$; (B) $20^{\circ} \mathrm{C}$ e de 23,9 a $48,2 \%$; (C) $30^{\circ} \mathrm{C}$ e de 23,3 a $49,3 \%$. 
$10^{\circ} \mathrm{C}$

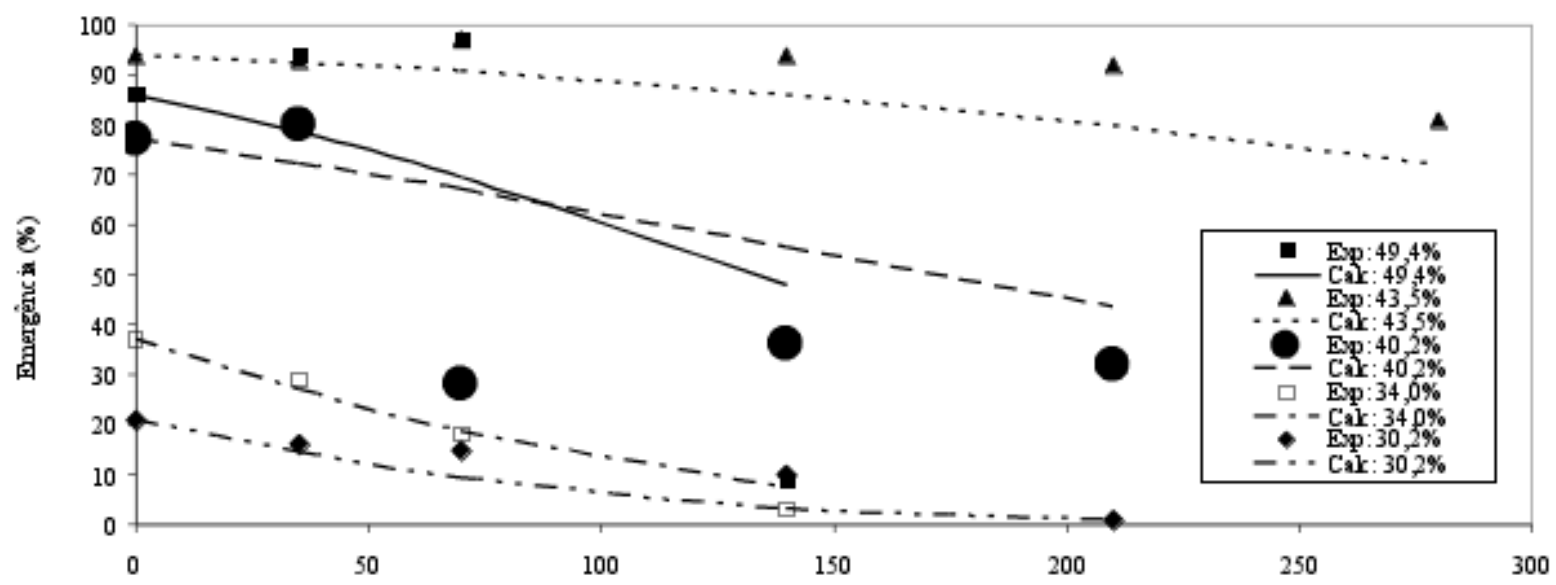

$20^{\circ} \mathrm{C}$

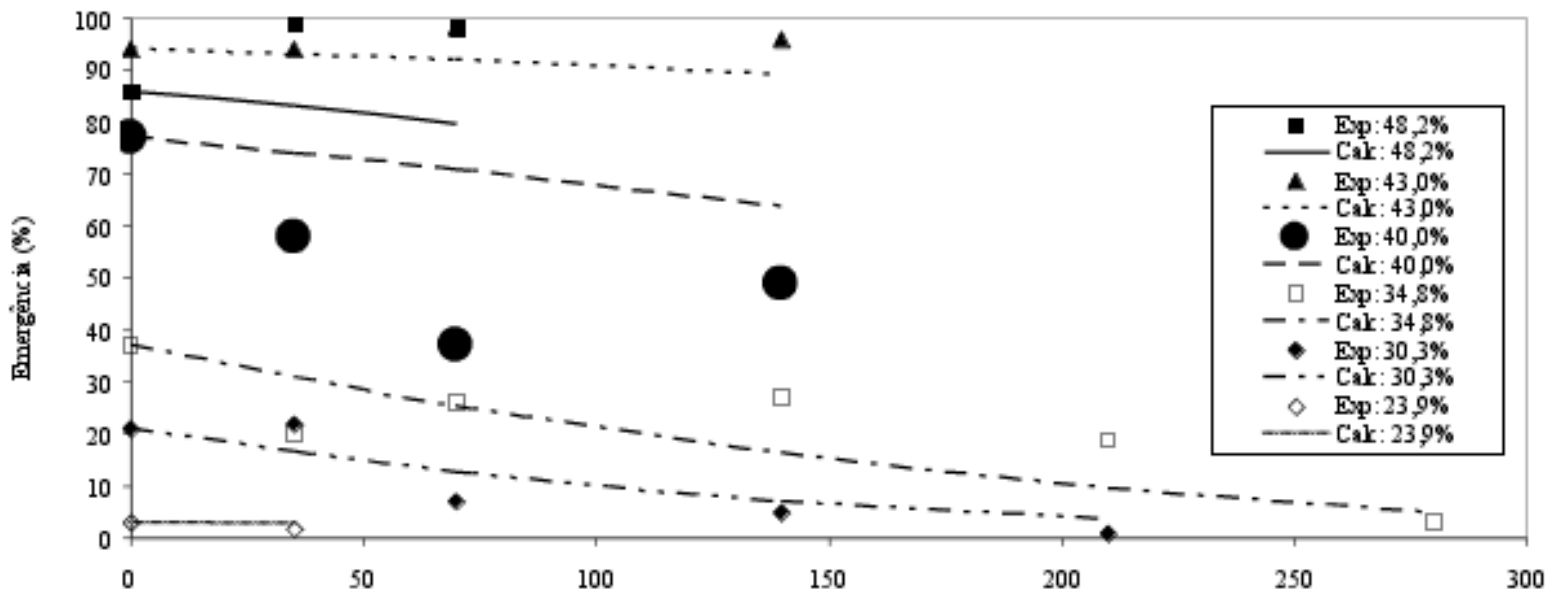

$30^{\circ} \mathrm{C}$

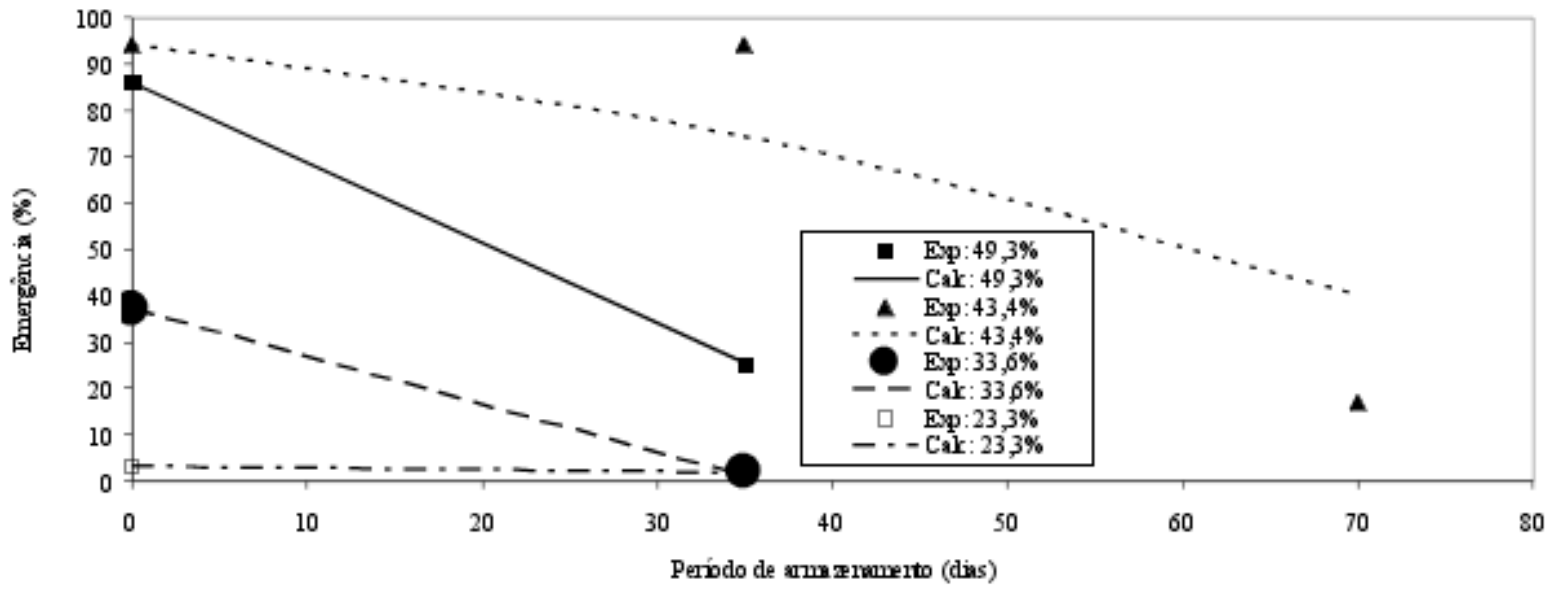

Figura 2. Valores experimentais e calculados da perda da emergência de sementes de camu-camu em função do período de armazenamento para três temperaturas e teores de água: (A) $10{ }^{\circ} \mathrm{C}$ e de 30,2 a 49,4\%; (B) $20{ }^{\circ} \mathrm{C}$ e de 23,9 a $48,2 \%$; (C) $30^{\circ} \mathrm{C}$ e de 23,3 a 49,3\%. 


\section{CONCLUSÕES}

- As equações recomendadas para descrever as perdas da germinação e emergência de sementes de camu-camu armazenadas, respectivamente, foram:

$$
\begin{aligned}
& \left.v=v_{0}-p / 10^{\left.\left(39,5,5693 \times 10^{-6} m^{3}-0,0055157 t\right)\right)}\right) \\
& v=v_{0}-p / 10^{\left.\left(29,8469-2,3751 \times 10^{-6} m^{3}-0,00633319 t\right)\right)}
\end{aligned}
$$

- As equações recomendadas foram ajustadas com razoável precisão para 57 observações experimentais, tanto para a perda da germinação $\left(\mathrm{R}^{2}\right.$ de 0,90 , d.p. de $12,3 \%$ e grau de significância dos coeficientes da equação menor que $0,4 \%$ de probabilidade de erro no teste $t$ ) como para a perda da emergência $\left(\mathrm{R}^{2}\right.$ de 0,87 , d.p. de $13,3 \%$ e grau de significância dos coeficientes da equação menor que $0,1 \%$ de probabilidade de erro no teste $t$ ).

\section{REFERÊNCIAS BIBLIOGRÁFICAS}

AFONSO JUNIOR, P.C.; CORRÊA, P.C.; QUEIROZ, D.M. Modelamento da perda de qualidade de sementes de soja, em função das condições iniciaise da atmosferano armazenamento. Revista Brasileira de Engenharia Agrícola e Ambiental, Campina Grande, v.4, n.3, p.403-408, 2000.

BLACK, M.; OBENDORF, R.L.; PRITCHARD, H.W. Damage and tolerance in retrospect and prospect. In: BLACK, M.; PRITCHARD, H.W. (Ed.). Desication and survival in plants: drying without dying. Wallingford: CABI, 2002. p.367382.

ELLIS, R.H.; ROBERTS, E.H. Improved equations for the prediction of seed longevity. Annals of Botany, Oxford, v.45, p.13-30, 1980.

ELLIS, R.H.; OSEI-BONSU, K.; ROBERTS, $\mathrm{H}$. The influence of genotype, temperature and moisture on seed longevity in chickpea, cowpea and soybean. Annals of Botany, Oxford, v.50, p.69-82, 1982.
FERREIRA, S.A.N.; GENTIL, D.F.O. Armazenamento de sementes de camu-camu (Myrciaria dubia) com diferentes graus de umidade e temperaturas. Revista Brasileira de Fruticultura, Jaboticabal, v.25, n.3, p.440-442, 2003.

GENTIL, D.F.O. Conservação de sementes de Myrciaria dubia (H. B. K.) McVaugh. 2003, 41. Tese (Doutorado em Agronomia/Fitotecnia). Escola Superior de Agricultura Luiz de Queiroz, Universidade de São Paulo, Piracicaba, 2003.

GENTIL, D.F.O.; FERREIRA, S.A.N. Armazenamento de sementes de camu-camu (Myrciaria dubia (H. B. K.) McVaugh). In: SEMINÁRIO ANUAL DE INICIAÇÃO CIENTÍFICA, 4., Manaus, 1991. Resumos... Manaus: UA/INPA, 1991. p.10.

GENTIL, D.F.O.; FERREIRA, S.A.N. Tolerância à dessecação e viabilidade de sementes de camucamu. Revista Brasileira de Fruticultura, Jaboticabal, v.22, n.2, p.264-267, 2000.

GENTIL, D.F.O.; SILVA, W.R.; FERREIRA, S.A.N. Conservação de sementes de camu-camu (Myrciaria dubia (H. B. K.) McVaugh). Bragantia, Campinas, v.63, n.3, p.421-430, 2004.

GINER, S.A.; LUPANO, C.E.; AÑON, M.C. A model for estimating loss of wheat seed viability during hot-air drying. Cereal Chemists, Inc., v.68, n.1, p-77-80, 1991.

HONG, T.D., LININGTON, S., ELLIS, R.H. Seed Storage Behaviour: a Compendium. Handbooks for Genebanks: No. 4. International Plant Genetic Resources Institute, Rome, 1996. 120p.

McVAUGH, R. Tropical american myrtaceae. Fieldiana - Botany, v.29, n.8, p-393-532, 1963.

MEDEIROS, A.C.S.; EIRA, M.T.S. Comportamento Fisiológico, Secagem e Armazenamento de Sementes Florestais Nativas. Embrapa Florestas, 2006. 13p. (Embrapa Florestas. Circular Técnica, 127). 
PRESS, W.H.; FLANNERY, B.P.; TEUKOLSKY, S.A.; VETTERLING, W.T. Numerical recipes. Cambridge University Press: Cambridge. 1990, 874p.

SACANDÉ, M.; JOKER, D.; DULLOO, M.E.; THOMSEN, K.A. Comparative storage biology of tropical tree seeds. Rome: IPGRI, 2004. 363p.

SAPRA, R.L.; NARAIM, P.; BHAT, S.R.; LAL, S.K.; JAIN, S.K. Prediction of seed longevity in the genebank: How reliable are the estimates? Current science, Bangalore, v.85, n.11, p.16121616, 2003.

SILVA, M.L. Avaliação da produção de mudas de camu-camu (Myrciaria dubia (H.B.K.) Mc Vaugh) por meio de estacas de diferentes diâmetros submetidos a concentrações do ácido naftaleno acético _ANA. Manaus, 2001. 60 f. Dissertação $($ mestrado $)=$ Instituto Nacional de Pesquisas da Amazônia, Universidade do Amazonas.

SINÍCIO, R. Equação de longevidade para sementes de café (Coffea arábica L.). Engenharia na Agricultura, Viçosa, v.19, n.4, p.287-297, 2011.

SINÍCIO, R. Equação de longevidade para sementes de jabuticaba (Myrciaria jaboticaba (Vell.) O. Berg). Engenharia na Agricultura, v.21, n.4,p.323-333, 2013.

SINÍCIO, R.; LOPES， J.F.; SILVA， D.J.H.;
MATTEDI, A.P. Longevity equation for tomato seeds. Seed Science and Technology, Zurich, v.37, n.3, p.667-675, 2009.

SINÍCIO, R.; MAURI, A.L.; ARAUJO, E.F. Equacionamento da longevidade de sementes de tomate (Lycopersicum esculentum Mill). Revista Brasileira de Armazenamento, Viçosa, v.33, n.2, p.127-133, 2008.

SOKHANSANJ, S. Through-flow dryers for agricultural crops. In: BAKER, C.G.J. (ed.) Industrial Drying of Foods. London: Blackie Academic \& Professional, 1997. 309p.

VILLANUEVA-TIBURCIO, J.E.; CONDEZOHOYOS, L.A.; ASQUIERI, E.R. Antocianinas, ácido ascórbico, polifenoles totales y actividad antioxidante, en la cáscara de camu-camu (Myrciaria dubia (H.B.K) McVaugh. Ciência e Tecnologia de Alimentos, Campinas, v.30(Supl.1): 151-160, 2010.

YUYAMA, K.; AGUIAR, J.P.L.; YUYAMA, L.K.O. Camu-camu: um fruto fantástico como fonte de vitamina C. Acta Amazônica, Manaus, v.32, n.1, p.169-174, 2002.

YUYAMA, K.; MENDES, N.B.; VALENTE, J.P. Longevidade de sementes de camu-camu submetidas a diferentes ambientes e formas de conservação. Revista Brasileira de Fruticultura, Jaboticabal, v.33, n.2, p.601-607, 2011. 